\title{
Missbrauch-Opfer: Therapie festigt die Erinnerung
}

Goodman GS et al. Psychological Counseling and Accuracy of Memory for Child Sexual Abuse. Am Psychol 2017; 72: 920-931

Traumatherapie kann die Erinnerung an ein traumatisches Ereignis verändern. Sind Kinder, die Opfer sexuellen Missbrauchs wurden, deshalb weniger zuverlässige Zeugen, wenn sie bereits in therapeutischer Behandlung waren? Muss eine Therapie ggf. bis nach Prozessende warten? Oder ist die Aussage der Kinder nach einer Therapie schlicht unbrauchbar? Eine US-Langzeitstudie hat jetzt Zeugenaussagen direkt nach dem Ereignis und 10-16 Jahre später verglichen.

Die Studienteilnehmer waren zwischen 1985 und 1987 an Strafprozessen beteiligt. Damals (Zeitpunkt 1) wurden die Aussagen der Kinder sowie andere Belege (Polizeiberichte, medizinische Untersuchungsergebnisse etc.) dokumentiert. 10-16 Jahre (Zeitpunkt 2) später wurden die Betroffenen erneut kontaktiert und befragt.

\section{Genauere Erinnerung nach Therapie}

Das Ergebnis war erstaunlich eindeutig: Studienteilnehmer, die zu Zeitpunkt 1 eine psychologische Beratung oder Therapie erhalten hatten, gaben zu Zeitpunkt 2 signifikant mehr richtige Antworten auf Fragen zu dem Missbrauch. Vor allem übertriebene Darstellungen des Vorfalls traten bei den ehemaligen Therapie-Nutzern seltener auf. Untertriebene Darstellungen waren in beiden Gruppen gleich häufig.

Die wahrscheinlichste Erklärung: In den therapeutischen Gesprächen wurden die Details des Missbrauchs besprochen und die Erinnerung an sie so gefestigt. Auch das erarbeitete Verständnis des Vorfalls (oder: der Vorfälle) hat vermutlich zu einer kohärenten Schilderung beigetragen, die die $\mathrm{Er}$ innerung festigt und erleichtert. Möglich ist aber auch, dass therapeutische Interventionen zu einer Symptomreduktion geführt und so die Erinnerung an das traumatische Ereignis erleichtert haben - Studien belegen jedenfalls, dass Betroffene mit weni-

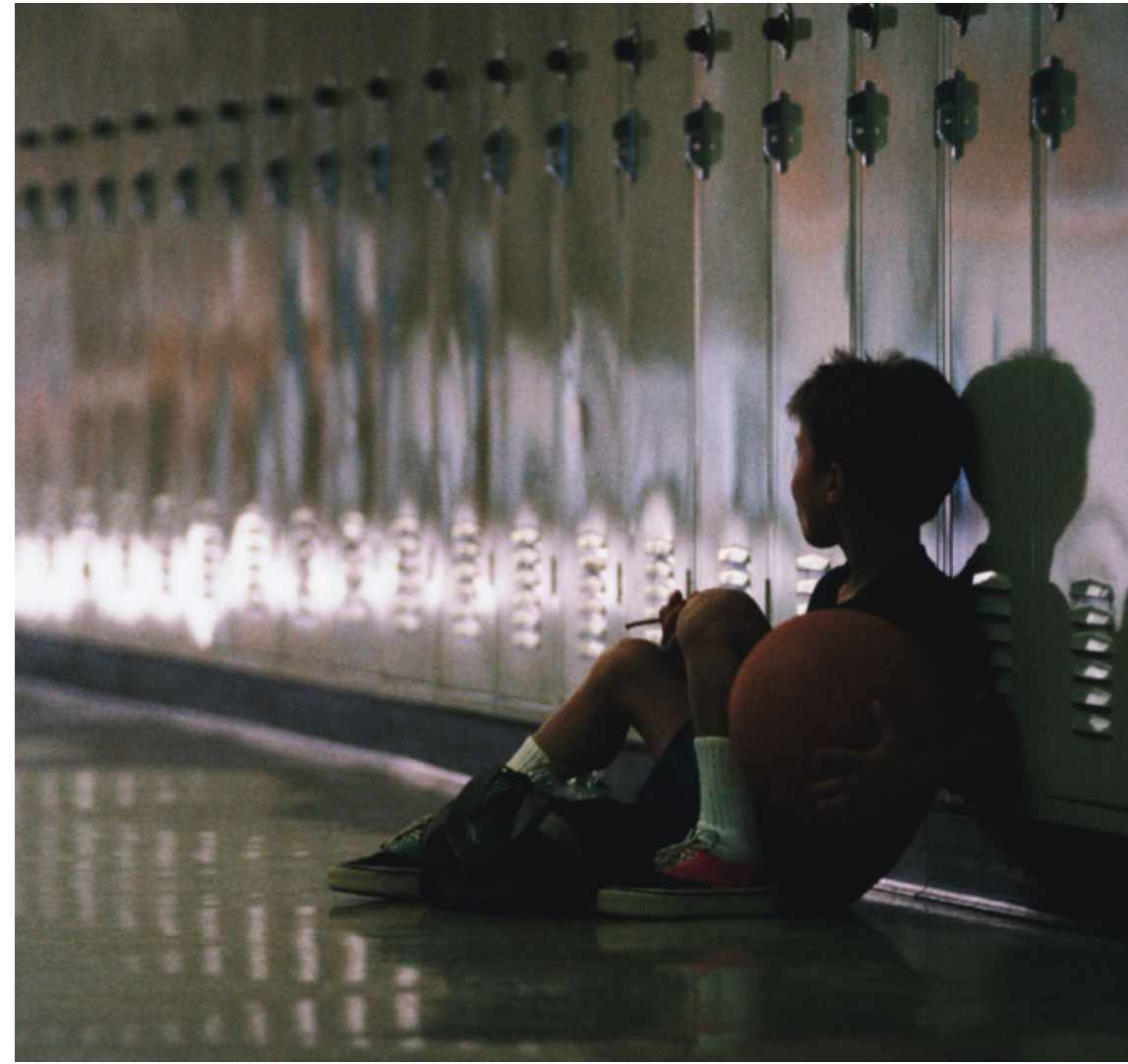

Missbrauch-Opfer sagen oftmals erst viele Jahre nach dem Ereignis als Zeugen aus. Beeinflusst eine vorangegangene Therapie dabei ihre Aussage? (Quelle: PhotoDisc)

ger psychopathologischen Symptomen sich i.d.R- besser erinnern.

\section{Zuverlässige Aussagen - auch Jahre später}

Die Autoren haben außerdem andere mögliche Faktoren berücksichtigt, wie die Schwere des Missbrauchs, die Beziehung des Opfers zum Täter, Symptome einer Posttraumatischen Belastungsstörung (PTSD), das Alter des Kindes und ob es eine Zeugenaussage vor Gericht machen musste. Aber keiner dieser Faktoren beeinflusste die Erinnerung der Kinder (später Jugendlichen oder jungen Erwachsenen) - nur eine therapeutische Intervention in der Akutphase.

Dieses Ergebnis ist insbesondere deshalb bedeutsam, weil Missbrauch-Opfer sich oft erst viele Jahre nach dem Ereignis zu Wort melden. Die Studie zeigt: Ihre Glaubwürdig- keit wird durch eine vorangegangene Therapie nicht beeinträchtigt.

FAZIT

Zwar handelt es sich um eine epidemiologische Erhebung; die Teilnehmer wurden nicht randomisiert einer Therapie zugeordnet. Kausale Zusammenhänge können daher nicht sicher belegt werden. Es spricht jedoch einiges dafür, dass eine frühe therapeutische Intervention weder den Betroffenen schadet noch ihrer Erinnerung an das Ereignis - vorausgesetzt, dass keine Techniken verwendet werden, die die Erinnerung aktiv manipulieren. Die gefestigte Erinnerung könnte auch Zeugenaussagen der Opfer zugutekommen.

Dr. Nina Drexelius, Hamburg 